The Angular Distribution of Particles Emerging from a Diffusive Region and its Implications for the Fleck-Canfield Random Walk Algorithm for Implicit Monte Carlo Radiation Transport

M. A. Cooper

July 3, 2000

Lawrence

Livermore

National

Laboratory

U.S. Department of Energy

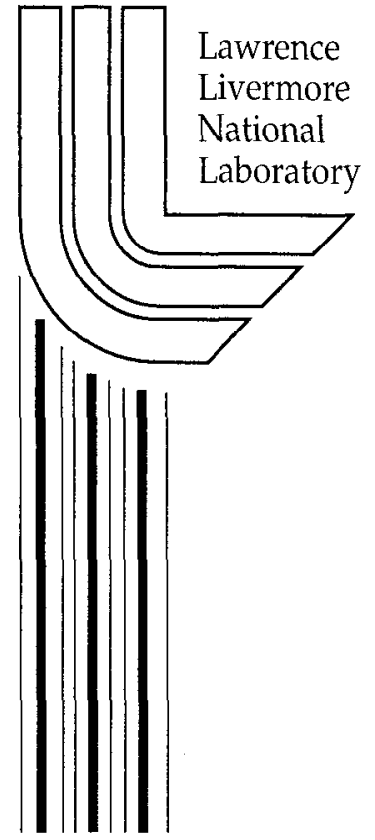




\section{DISCLAIMER}

This document was prepared as an account of work sponsored by an agency of the United States Government. Neither the United States Government nor the University of California nor any of their employees, makes any warranty, express or implied, or assumes any legal liability or responsibility for the accuracy, completeness, or usefulness of any information, apparatus, product, or process disclosed, or represents that its use would not infringe privately owned rights. Reference herein to any specific commercial product, process, or service by trade name, trademark, manufacturer, or otherwise, does not necessarily constitute or imply its endorsement, recommendation, or favoring by the United States Government or the University of California. The views and opinions of authors expressed herein do not necessarily state or reflect those of the United States Government or the University of California, and shall not be used for advertising or product endorsement purposes.

Work performed under the auspices of the U.S. Department of Energy by the University of California Lawrence Livermore National Laboratory under Contract W-7405-Eng-48.

This report has been reproduced directly from the best available copy.

Available to DOE and DOE contractors from the Office of Scientific and Technical Information

P.O. Box 62, Oak Ridge, TN 37831

Prices available from (423) 576-8401

http://apollo.osti.gov/bridge/

$\Lambda$ vailable to the public from the

National Technical Information Service

U.S. Department of Commerce

5285 Port Royal Rd.,

Springficld, VA 22161

http://www.ntis.gov/

OR

Lawrence Livermore National Laboratory

Technical Information Department's Digital Library

http://www.llnl.gov/tid/Library.html 


\title{
The Angular Distribution of Particles Emerging from a Diffusive Region and its Implications for the Fleck-Canfield Random Walk Algorithm for Implicit Monte Carlo Radiation Transport
}

\author{
Marc A. Cooper \\ Lawrence Livermore National Laboratory, L-018 \\ Livermore, CA 94550 USA \\ cooper38@llnl.gov
}

\begin{abstract}
We present various approximations for the angular distribution of particles emerging from an optically thick, purely isotropically scattering region into a vacuum. Our motivation is to use such a distribution for the Fleck-Canfield random walk method [1] for implicit Monte Carlo (IMC) [2] radiation transport problems. We demonstrate that the cosine distribution recommended in the original random walk paper [1] is a poor approximation to the angular distribution predicted by transport theory. Then we examine other approximations that more closely match the transport angular distribution.
\end{abstract}

\section{Introduction}

In the Fleck-Canfield random walk algorithm for IMC radiation transport problcms, in optically thick, highly scattering media, Monte Carlo transport is suspended and diffusion theory is used to transport particles. In this algorithm, the largest sphere that will fit in a given zone, centered at the current particle location, is inscribed. 'The classical diffusion equation is solved and probabilities are computed to determine if the particle escapes from the sphere. If it does escape, then the cosine of the polar angle (henceforth referred to as the angle) about the local outward normal to the location on the surface of the sphere from which the particle emerges must be computed. Fleck and Canfield recommend the use of a cosine distribution. As we show, this is not a good approximation. 
We show a simple method of deriving a more accurate angular distribution. By using two approximations for the extrapolated endpoint boundary condition, we generate two similar distributions. We also report results from numerical experiments performed by Bateson [3]. We see that Bateson's result matches that obtained using the diffusion extrapolation distance and that it is a very good approximation to transport theory.

This paper is organized in the following manner. Wc cxamine the transport angular distribution as derived by Chandrasekhar [4] in Section 2. We present the cosine distribution in Section 3. In Section 4, we derive two approximations using the extrapolated endpoint boundary condition. Bateson's results are presented in Section 5. In Section 6 we compare all of the distributions presented. We draw some conclusions in Section 7.

\section{The Transport Angular Distribution}

The problem of determining the angular distribution of particles emerging from a diffusive spherical region in the Fleck-Canfield random walk algorithm is analogous to the law of darkening in astrophysics [4] and the Milne problem in nuclear engineering [5] under certain assumptions. The law of darkening and Milne problems assume a semi-infinite half-space, with no incident flux on a vacuum boundary at $x=0$ and some source at infinity. For the Fleck-Canfield situation, if the sphere is optically thick so that the assumption that diffusion theory holds (that the specific intensity is nearly isotropic [6]) is reasonable, then it approaches the law of darkening/Milne problem.

The angular distribution we seek is a probability density function (pdf) [7] such that:

$$
p d f(\mu)=\text { const } \mu I(0, \mu), \quad 0<\mu \leq 1 .
$$

For the law of darkening, for a purely isotropically scattering medium, Chandrasekhar obtained an analytic transport result of

$$
p d f(\mu)=\frac{\sqrt{3}}{2} \mu H(\mu) .
$$

$H(\mu)$ is the Chandrasekhar $\mathrm{H}$-function defined as

$$
H(\mu)=\frac{1}{\mu_{1} \cdots \mu_{n}} \frac{\prod_{i=1}^{n}\left(\mu+\mu_{i}\right)}{\prod_{j=1}^{n}\left(1+k_{j} \mu\right)},
$$

where the $\mu_{i}$ 's are the zeros of the Legendre polynomial $P_{2 n}(\mu)$ and $k_{j}$ 's are the non-negative roots of some characteristic equation of form

$$
1=2 \sum_{m=1}^{n} \frac{a_{m} \Psi\left(\mu_{m}\right)}{1-k^{2} \mu_{m}^{2}},
$$


and $\Psi(\mu)$ is an even polynomial in $\mu$ that satisfies

$$
\int_{0}^{1} d \mu \Psi(\mu) \leq 0.5
$$

The H-function can be approximated as the solution of the following integral equation-

$$
H(\mu)=1+\mu H(\mu) \int_{0}^{1} d \mu^{\prime} \frac{\Psi\left(\mu^{\prime}\right)}{\mu+\mu^{\prime}} H\left(\mu^{\prime}\right) .
$$

For a purely isotropically scattering medium

$$
\Psi(\mu)=0.5
$$

Values of the H-function were determined numerically and tabulated in Table XI of Reference 4.

We also have the result [4]

$$
\int_{0}^{1} d \mu \mu H(\mu)=\frac{2}{\sqrt{3}}
$$

so we see that the pdf, Equation 2, is normalized.

\section{The Cosine Distribution}

The angular distribution of particles emitted from an isotropic surface source is described by a cosine distribution. The cosine pdf is

$$
p d f(\mu)=2 \mu
$$

We note that this is not an approximation of the transport result. This distribution arises from a different physical problem than the one we seek. Thus, as we show later in this paper, the cosine distribution is not accurate and should not be used in the Fleck-Canfield random walk algorithm. 


\section{Two Approximations}

In this section, we show two approximations to the transport angular distribution. These were derived by Larsen [7]. We begin by invoking the $P_{1}$ approximation

$$
I(x, \mu) \approx \frac{1}{2} I_{0}(x)+\frac{3}{2} \mu I_{1}(x)
$$

where

$$
I_{0}(x)=\int_{-1}^{1} d \mu I(x, \mu)
$$

and

$$
I_{1}(x)=\int_{-1}^{1} d \mu \mu I(x, \mu)
$$

Using Fick's law

$$
I_{1}(x)=-\frac{1}{3 \sigma} \frac{d I_{0}}{d x}
$$

we substitute $I_{1}(x)$ into Equation 10 to obtain

$$
I(x, \mu)=\frac{1}{2} I_{0}(x)-\frac{1}{2} \frac{\mu}{\sigma} \frac{d I_{0}}{d x} .
$$

In diffusion theory, a vacuum boundary can be modeled by setting $I_{0}$ to zero at an extrapolated boundary [8]

$$
I_{0}\left(x_{b}+d\right)=0
$$

where $x_{b}$ is the physical boundary and $d$ is the extrapolation distance. If we expand Equation 15 in a Taylor series we obtain

$$
I_{0}\left(x_{b}\right)+d \frac{d I_{0}\left(x_{b}\right)}{d x}=0
$$

Setting $x_{b}=0$, solving for the derivative term in Equation 16 and substituting this into Equation 14, which is evaluated at $x=0$, we have

$$
I(0, \mu)=\frac{1}{2} I_{0}(0)+\frac{1}{2} \frac{\mu}{\sigma d} I_{0}(0) .
$$


If we multiply Equation 17 by $\mu$, we obtain

$$
\mu I(0, \mu)=\frac{1}{2} I_{0}(0)\left[\mu+\frac{1}{\sigma d} \mu^{2}\right] .
$$

Comparing this to Equation 1, we write the pdf as

$$
p d f=a\left(\mu+\frac{1}{\sigma d} \mu^{2}\right) .
$$

This pdf, as is the case for all pdf's, must integrate to unity. We do this to obtain an expression for $a$

$$
a=\left(\frac{1}{2}+\frac{1}{3 \sigma d}\right)^{-1} .
$$

Now our pdf is

$$
p d f=\left(\mu+\frac{1}{\sigma d} \mu^{2}\right)\left(\frac{1}{2}+\frac{1}{3 \sigma d}\right)^{-1}
$$

Our two approximations concern the value of $d$, the extrapolation distance. We call the Marshak approximation one in which

$$
d=\frac{2}{3 \sigma}
$$

yields the Marshak boundary condition for a vacuum boundary [6]. Thus,

$$
p d f=\mu+\frac{3}{2} \mu^{2} .
$$

We call the Milne approximation one in which

$$
d=\frac{0.7104}{\sigma}
$$

is the Milne extrapolation distance [5]. So,

$$
p d f=1.03176 \mu+1.45236 \mu^{2} .
$$

\section{Bateson's Results}

Bateson [3] considered a finite, purely isotropically scattering slab, with vacuum boundaries on the left and right, in which particles were emitted isotropically from the center. He ran 
Monte Carlo simulations and examined the angular distribution of particles that escaped an optically thick slab at late times. Thus he considered particles that had achieved an asymptotic distribution. He plotted his results and used a curve fit to obtain

$$
p d f=\mu+\frac{3}{2} \mu^{2}
$$

This is the same pdf that was derived in Section 4 for the Marshak distribution, Equation 23. Since a curve fit would not yield exactly 1 and 1.5 for the coefficients of $\mu$ and $\mu^{2}$ respectively, it is assumed that Bateson obtained coefficients close to these values and then realized that rounding the values yielded a normalized distribution.

\section{Comparison}

Now we compare the four distributions considered: Chandrasekhar H-function, cosine, Marshak, and Milne. Looking at Figure 1, we immediately notice how poor the cosine approximation is. We also note how closely the Chandrasekhar H-function, Marshak, and Milne distributions match.

Let us examine the average angle of each distribution, defined as

$$
\bar{\mu}=\frac{\int_{0}^{1} d \mu \mu p d f(\mu)}{\int_{0}^{1} d \mu p d f(\mu)} .
$$

Values for the various distributions are shown in Table 1.

Again, we see that the cosine distribution is not accurate and the other distributions yield similar results. From close examination of Figure 1 and from the average angles in Table 1, it is apparent that the Marshak result is closer to the transport distribution (the Chandrasekhar $\mathrm{H}$-function) than the Milne result. This was not anticipated.

First, we must acknowledge that the Chandrasekhar result is a numerical solution of an approximation, Fquation 6, to the H-function that is obtained from transport theory, Equation 3. However, an exact analytical expression can be obtained for the integral of the H-function [4]

$$
\int_{0}^{1} d \mu H(\mu)=\frac{2}{\varpi_{0}}\left[1-\left(1-\varpi_{0}\right)^{\frac{1}{2}}\right]
$$


In our case of purely isotropic scattering, we have $\varpi_{0}=1$ and

$$
\int_{0}^{1} d \mu H(\mu)=2 .
$$

As shown in Chapter V, Table XII of Reference 4, the numerical procedure used yields a result of 1.9999 for the integral of the $\mathrm{H}$-function. Thus, the tabulated $\mathrm{H}$-function results are very accurate, and the Chandrasekhar distribution can be considered a very good representation of the transport distribution.

Since the Milne extrapolation distance is more accurate than the Marshak distance, we would expect that the Milne distribution would be more accurate than the Marshak distribution. We would also expect that Bateson's Monte Carlo simulations would match the Milne rather than the Marshak results. Nevertheless, it is clear that the Marshak angular distribution is a more accurate representation of the transport distribution than the Milne approximation.

\section{Conclusion}

We have examined various expressions for the angular distribution of particles emerging from a diffusive region. The motivation for considering such problems was to find an accurate expression for the angular distribution of Monte Carlo particles that escape from a diffusive sphere in the Fleck-Canfield random walk algorithm for IMC radiation transport. If the sphere is optically thick and the scattering ratio is near unity, this problem is analogous to the law of darkening/Milne problem for isotropic scattering.

Chandrasckhar [4] has derived the transport result for the angular distribution. Idcally, we would like to use this in the Fleck-Canfield method, but the resulting pdf involves a function, the $\mathrm{H}$-function, that does not have an analytical solution. Although it has been tabulated, we seek an analytical expression that is accurate and can be readily inverted for use in the Fleck-Canfield method.

We demonstrated that the Marshak distribution is an accurate representation of the transport distribution. Use in the Fleck-Canfield method requires that we invert the pdf, Equation 23. Although it involves solving a cubic equation, this can be done quickly with the Newton-Raphson method [9]. The Marshak result was obtained analytically by Larsen [7] using the " $P_{1}$ " approximation and the Marshak boundary condition for a vacuum boundary. 
Interestingly, this yielded the same result as that obtained by Bateson [3] via Monte Carlo simulations.

We conclude that the Marshak angular distribution, Equation 23, should be used in the Fleck-Canfield method instead of the cosine distribution as recommended in Reference 1.

\section{References}

[1] J.A. Fleck and E.H. Canfield, "A Random Walk Procedure for Improving the Computational Efficiency of the Implicit Monte Carlo Method for Nonlinear Radiation Transport," Journal of Computational Physics 54, 508-523 (1984).

[2] J.A. Fleck and J.D. Cummings, "An Implicit Monte Carlo Scheme for Calculating Time and Frequency Dependent Nonlinear Radiation Transport," Journal of Computational Physics 8, 313-342 (1971).

[3] W.B. Bateson, "Recovering Transport from Diffusion", Lawrence Livermore National Laboratory, unpublished (1999).

[4] S. Chandrasekhar, Radiative Transfer, Dover Publications, Inc., New York (1960).

[5] K.M. Case and P.F. Zweifel, Linear Transport Theory, Addison-Wesley Publishing Company, Inc., Reading (1967).

[6] G.C. Pomraning, 'The Equations of Radiation Hydrodynamics, Pergamon Press, Oxford (1973).

[7] E.W. Larsen, University of Michigan, private communication (2000).

[8] J.J. Duderstadt and L.J. Hamilton, Nuclear Reactor Analysis, John Wiley \& Sons, Inc., New York (1976).

[9] W.H. Press, S.A. Teukolsky, W.T. Vetterling, and B.P. Flannery, Numerical Recipes in C, Cambridge University Press, Cambridge (1997).

This work was performed under the auspices of the U.S. Department of Energy by the University of California Lawrence Livermore National Laboratory under contract No. W-7405-Eng-48. 


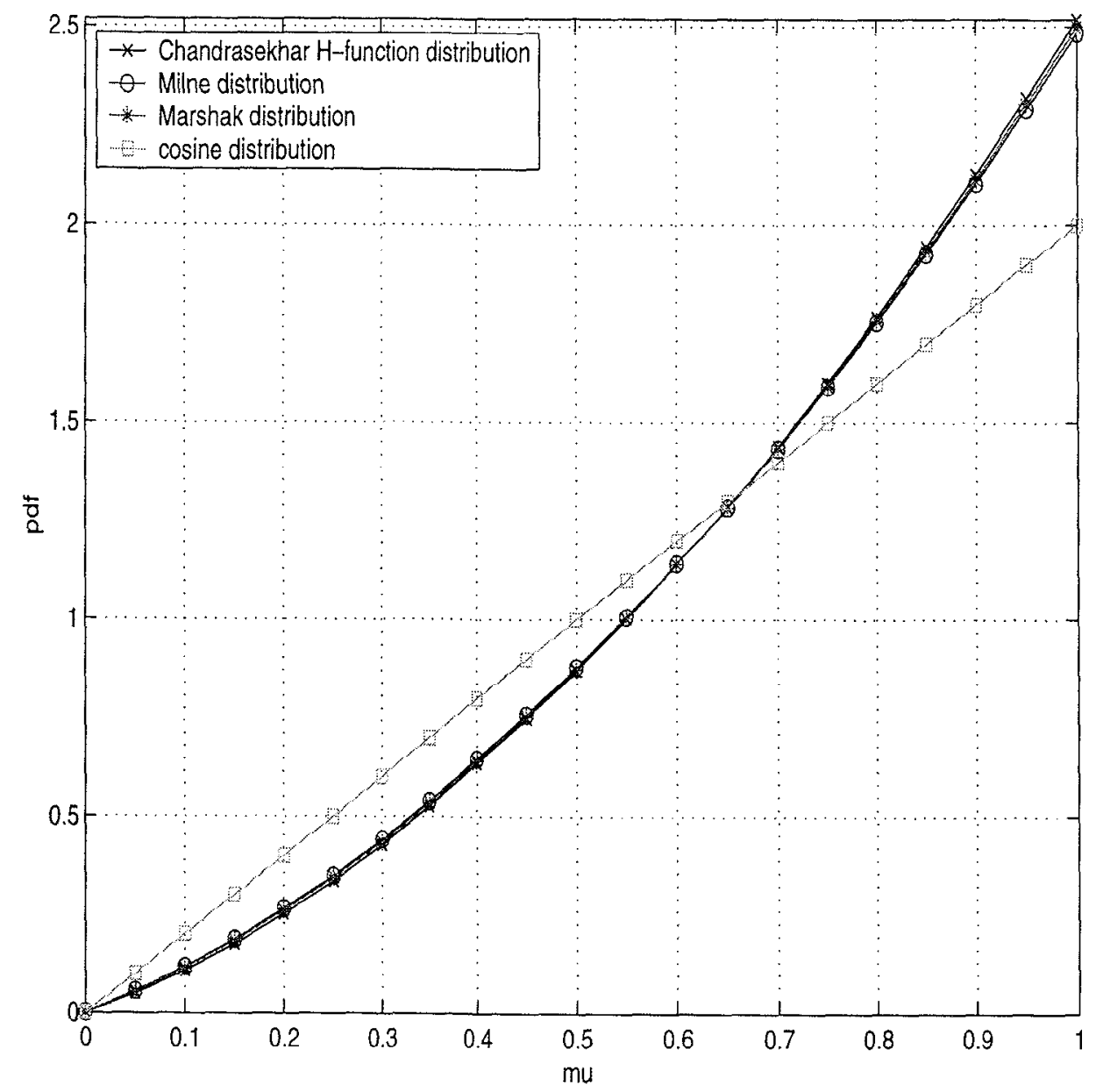

Figure 1: Angular Distributions.

\begin{tabular}{|c|c|}
\hline Distribution & $\bar{\mu}$ \\
\hline \hline Chandrasekhar & 0.71045 \\
\hline Marshak & 0.70833 \\
\hline Milne & 0.70701 \\
\hline cosine & 0.66667 \\
\hline
\end{tabular}

Table 1: Average Angles. 